
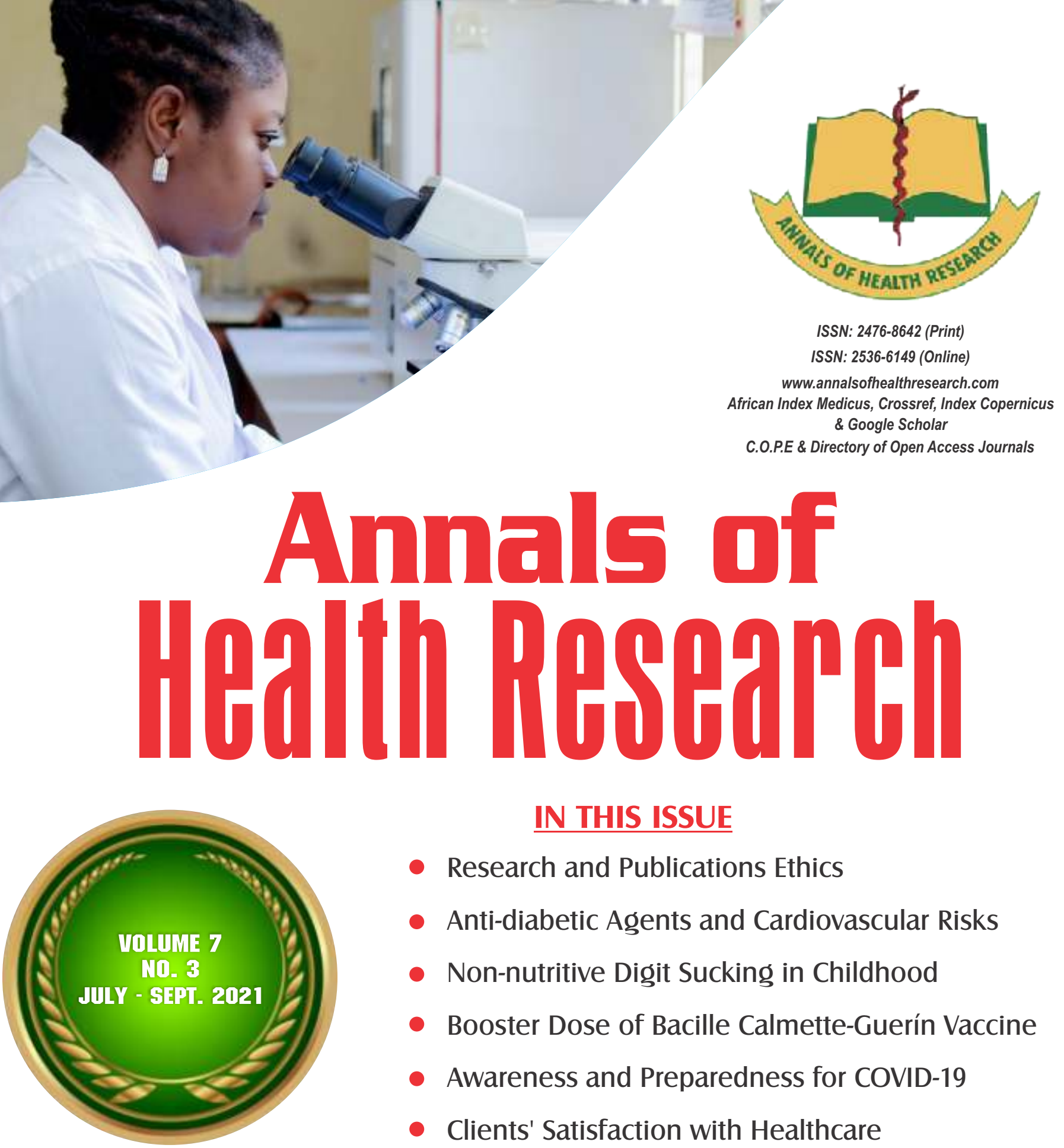

IN THIS ISSUE

- Research and Publications Ethics

- Anti-diabetic Agents and Cardiovascular Risks

- Non-nutritive Digit Sucking in Childhood

- Booster Dose of Bacille Calmette-Guerín Vaccine

- Awareness and Preparedness for COVID-19

- Clients' Satisfaction with Healthcare

- Antimicrobial Activity of Lactic Acid Bacteria

- Saddle Block for Transrectal Prostate Biopsy

- Physical and Mental Health of Stroke Survivors

- Transfusion Transmissible Infections

- Cervical Ectopic Pregnancy

PUBLISHED BY THE MEDICAL

AND DENTAL CONSULTANTS ASSOCIATION

OF NIGERIA, OOUTH, SAGAMU, NIGERIA.

www.mdcan.oouth.org.ng 


\title{
Anti-diabetic Agents and the Potentials for Reducing Cardiovascular Risks in Type-2 Diabetes Mellitus Ezeani IU*1, Eregie A ${ }^{2}$, Ohwovoriole EA ${ }^{3}$
}

\author{
1Endocrinology, Diabetes and Metabolism Unit, Department of Medicine, Federal Medical Centre, PMB 7001, \\ Umuahia, Abia State, Nigeria \\ 2Endocrinology, Diabetes and Metabolism Unit, Department of Medicine, University of Benin Teaching Hospital, \\ PMB 1111, Benin city, Edo State, Nigeria \\ ${ }^{3}$ College of Medicine, Bingham University, Karu, Plateau State, Nigeria
}

*Correspondence: Dr IU Ezeani, Department of Medicine, Federal Medical Centre, P.M.B 7001, Umuahia, Abia State, Nigeria. E-mail: ignatiusez@yahoo.com;

ORCID - https://orcid.org/0000-0002-6147-3106.

Summary

Recent reports from Cardiovascular Outcome Trials (CVOTs) revealed that some newer anti-diabetic drugs impact Major Adverse Cardiovascular Events (MACE). These medications include the Sodium-Glucose Co-Transporter (SGLT2) inhibitors and the Glucagon-like Peptide-1 (GLP-1) receptor agonists. There is a need for a review of the mechanisms of action of these drugs, in addition to their glucose-lowering effects and CV benefits. This review paper aims to explore the cardio-protective effects and CV risks of anti-diabetic medications, their mechanisms of action and the CV benefits evidenced by CVOTs. Using internet search, with search items such as Type 2 Diabetes mellitus, cardiovascular risk factors, cardiovascular outcome trials, major adverse cardiovascular events, sodium-glucose transporter-2 inhibitors, glucagon-like peptide-1 receptor agonist, the Google Scholar, EMBASE, PubMed, Medline, Web MD, and Scopus were checked for various relevant published articles. Analyses of the results of multiple CVOTs from various parts of the world were considered. These CVOTs were reviewed to assess the role of anti-diabetic agents in reducing cardiovascular risk in patients with T2DM. The SGLT2 inhibitors and GLP1 agonists were found to be beneficial in lowering MACE when compared with placebo. This is in addition to their anti-hyperglycaemic benefits. In conclusion, SGLT2 inhibitors and GLP-1 agonists confer dramatic beneficial CV risk reduction on patients with T2DM, as shown by the various CVOTs. This is in addition to their anti-hyperglycaemic effects. This remarkable benefit justifies the need by various guidelines to adopt them as second line agents to metformin in managing patients with T2DM.

Keywords: Anti-diabetic drugs, Cardiovascular risks, GLP1 agonist, Outcome trials, SGLT2 inhibitors.

\section{Introduction}

According to the World Health Organization (WHO), the number of people with Diabetes mellitus (DM) rose from 108 million in 1980 to 422 million in 2014. ${ }^{[1]}$ The prevalence of this disease among adults was $4.7 \%$ in 1980 , and it increased to $8.5 \%$ in 2014 . The majority of the people with DM have Type 2 Diabetes (T2DM). [2] Estimates from 255 high-quality data sources indicate that 
in 2045, approximately 700 million people will have diabetes mellitus. [1] The prevalence of T2DM continues to rise worldwide, despite the many efforts and lifestyle interventions adopted to help reduce its incidence. ${ }^{[3-5]} \mathrm{DM}$ is associated with a two to four-fold increase in the risk of coronary heart disease (CHD). [6,7] On its own, clinically established CHD is related to an increase in mortality by three to sevenfold. ${ }^{[8]}$ In a study by Haffner et al.,[9] a comparison of the seven-year incidence of myocardial infarction (MI) among diabetics and non-diabetics was made. It was demonstrated that the incidence of non-diabetic patients with or without $\mathrm{MI}$ at baseline were $18.8 \%$ and $3.5 \%$, respectively. In contrast, the incidence of $\mathrm{MI}$ in diabetic patients with or without MI at baseline was $45.0 \%$ and $20.2 \%$, respectively. From this study, it can be surmised that diabetic patients without previous MI have the same risk of MI as non-diabetic patients with previous MI. [9]

In the WHO Multinational Study of Vascular Disease in Diabetes (WHO MSVDD), which was initiated in 1974, a total of 4,713 deaths in T2DM patients were followed up to establish the cause of death. Cardiovascular disease was the most common underlying cause of death, accounting for $52 \%$ of the deaths. [10] In addition to acute cardiac events, the incidence of heart failure (HF) was higher in the diabetic population when compared to those without diabetes. [11] Despite the major contribution of CVD to morbidity and mortality in T2DM, earlier studies looking at the impact of glycaemic control on CVD and related mortality revealed no or modest impact. Indeed, the CVD risk reduction benefit seems to be derived from the appropriate care of the traditional CVD risk factors, including hypertension, dyslipidaemia, and obesity. [12,13]

In the management of DM, it is crucial to achieve reasonable glycaemic control and control the risk for CVD. This can be achieved by using an individualised, patient-centred approach to care.
[14,15] The traditional drugs that have been in use for T2DM treatment have not shown the desired benefit of reducing the CVD risk in these patients. In a meta-analysis of 42 trials, rosiglitazone demonstrated an increased risk of MI by $43 \%$ and cardiovascular mortality by $64 \%$ compared to other anti-diabetic drugs. [16] On the other hand, pioglitazone use for T2DM treatment has proven $\mathrm{CV}$ benefit. During a large clinical study, it significantly lowered the incidence of three-point MACE, including nonfatal MI, nonfatal stroke, and $\mathrm{CV}$ death in diabetic, pre-diabetic and insulin-resistant patients with established CVD. [17] However, pioglitazone increases the risk of $\mathrm{HF}$, bone fracture, weight gain and oedema. Therefore, its benefits must be weighed against its risks. [18]

Another class of drugs that has been in use for many decades to treat T2DM is sulphonylureas. They have also been associated with increased $\mathrm{CV}$ risk, $\mathrm{CV}$ death and all-cause mortality. In a cohort of 9,4750 patients, 17612 were initiated on sulphonylureas and 77138 on metformin. This study demonstrated that patients started on sulphonylurea had higher $\mathrm{CV}$ events compared to those commenced on metformin. ${ }^{[19]}$ A metaanalysis of 33 studies showed that sulphonylureas increased composite CV events by $10 \%$. [20]

A recent report from CVOTs revealed that some newer anti-diabetic drugs had an impact on MACE. [21] These medications include the SGLT2 inhibitors and the GLP-1 receptor agonists. The European Union approved the first set of SGLT2 inhibitors in 2012 and the FDA in 2014 to lower blood glucose levels in adults with T2DM. The drugs in this class include canagliflozin, dapagliflozin, and empagliflozin. [22] These new oral drugs have become good options in the management of DM with CVD. Among the novel class, SGLT-2 inhibitors were found to be helpful in patients with chronic HF. Empagliflozin and canagliflozin were found to reduce the risk of $\mathrm{CV}$ 
mortality, nonfatal MI and stroke in patients with DM and CVD. [22] GLP-1 analogues have been approved for use in the treatment of T2DM. The first drug in this class to be approved in the United States in 2005 was exenatide. [23] Others include lixisenatide, liraglutide, albiglutide, dulaglutide, and semaglutide. According to ADA and most guidelines, metformin is the preferred first-line therapy for T2DM, but GLP-1 analogues are considered in patients intolerant of metformin. ${ }^{24]}$ To the best of our knowledge, no extensive literature review of the major CVOTs highlighting their strengths and limitations have been carried out in our clime. This review will explore the cardio-protective effects and CV risks of oral anti-diabetic drugs used in patients with $\mathrm{DM}$, their mechanism of action and the $\mathrm{CV}$ benefits evidenced by CVOTs.

\section{SGLT2 INHIBITORS}

The SGLT2 inhibitors work by their preferential blockade of the SGLT2 transporter receptor in the S1 segment of the proximal convoluted tubules. This transporter is responsible for the reabsorption of $>90 \%$ of filtered glucose, thus resulting in increased glucose excretion. [25] The kidneys reabsorb glucose filtered by the glomerulus, and the threshold for reabsorption is equivalent to a blood glucose concentration of $10 \mathrm{mmol} / \mathrm{L}$. ${ }^{[26]}$ This threshold increases in people with T2DM, worsening the existing hyperglycaemic state. The SGLT2 inhibitors, by blocking this process, will lead to renal glucosuria, with a resultant decrease in blood glucose, without causing hypoglycaemia. [27] The diagram below illustrates the mechanism of action of these agents. [28]

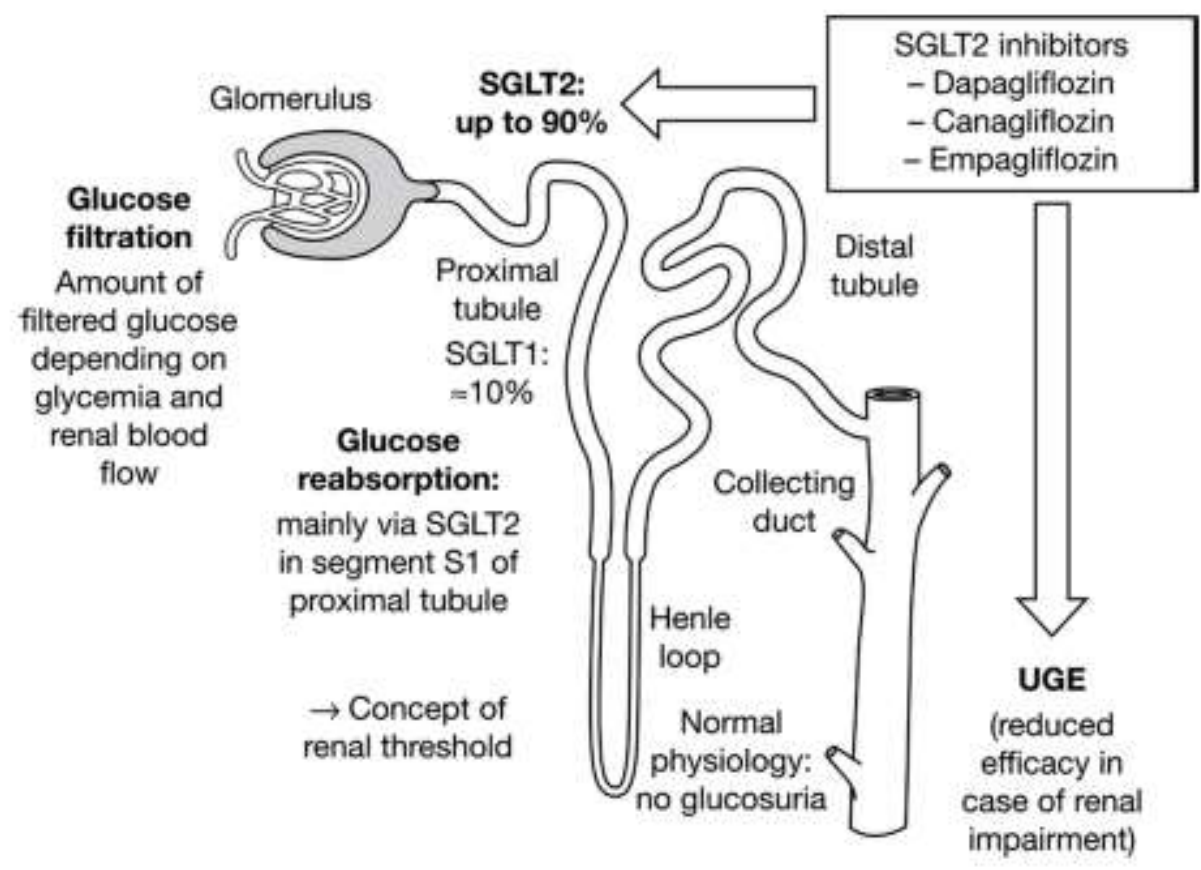

Figure 1: Mechanism of action of SGLT2 inhibitors.

Cornell, S. 'Mode of action of SGLT2 inhibitors in the kidney', 2015. Available at: https://www.researchgate.net/figure/Mode-of-actionof-SGLT2-inhibitors-in-the-kidney-Copied-with-permissionfrom-Scheen-12_fig1_283449175 (Accessed 22 July 2021).

Physiologic mechanism for the cardiovascular protecting effect of SGLT2 inhibitors
The mechanisms behind the $\mathrm{CV}$ benefits seen with these agents are unclear but appear to be independent of their glucose-lowering 
properties. [29] It is well known that SGLT2 inhibitors induce osmotic diuresis, which causes a reduction in the pre-load and after-load, resulting in blood pressure decrease and improved ventricular function. Also, there is an associated decrease in ventricular loading with enhanced cardiac function. Another mechanism is by inhibiting the sodium/hydrogen exchange in the myocardial cells, which reduces cytoplasmic sodium and calcium levels while increasing mitochondrial calcium levels, thus restoring sodium homeostasis. [30] This is the direct opposite of what we have in HF, where there is an increase in the cytosolic sodium and calcium.

Also, SGLT2 inhibitors reduce that rate of necrosis and fibrosis of cardiac muscles: preliminary studies have shown that dapagliflozin exhibits an antifibrotic effect by suppressing collagen production through the activation of M2 macrophages and inhibition of myofibroblast differentiation. [30] Similarly, empagliflozin can reduce adverse remodelling and HF in a porcine model by improving cardiac function. [31] It is noteworthy to mention that perivascular and epicardial fat has been implicated in the pathogenesis of HF in DM, and SGLT2 inhibitors help reduce fat accumulation and inflammatory activity. ${ }^{[32]}$ Additionally, they lower leptin levels which may have a role in cardiac inflammation and fibrosis. [30] Interestingly, they are known to slightly increase ketone and beta-hydroxybutyrate production, which offers an alternative myocardial fuel for those with diabetes. [30] This is used as a super fuel oxidised by the heart in preference to glucose and non-esterified fatty acids, thus helping to improve the mechanical efficiency of the heart. It is well known that Ischaemia/reperfusion injury can lead to the death of cardiac myocytes and HF. Recent data suggest that SGLT2 inhibition has a cardio-protective effect against ischaemia/reperfusion injury in diabetic and non-diabetic rats. [30] This beneficial effect of
SGLT2 inhibition is associated with a reduction in calmodulin kinase II activity, resulting in improved sarcoplasmic reticulum Ca2 $\mathrm{p}$ flux and increased contractility. However, it remains unclear if this effect also occurs in humans.

Remarkably, SGLT2 inhibitors lower plasma uric acid levels, which adversely affect the prognosis of HF. [33] Therefore, the use of these agents has been associated with small reductions in uric acid levels, which may be attributed to increased glycosuria in the proximal tubules due to SGLT2 inhibition, which stimulates uric acid secretion. [33] However, whether a reduction in hyperuricaemia by SGLT2 inhibition is a marker or it plays a causal role remains unknown. Further, it is well known that cardiac autophagy and degradation of lysosomes can be impaired in DM and HF. [34] Therefore, it has been postulated that SGLT2 inhibition can promote autophagy and lysosomal degradation by stimulating catabolic rates due to sustained glycosuria. [34] This leads to improved mitochondrial morphology and function. Therefore, it cannot be ruled out that some of the benefits of SGLT2 inhibition in heart failure may be secondary to their effects on stimulating autophagy. They can increase haematocrit levels due to their ability to promote erythropoiesis through enhanced erythropoietin (EPO) secretion by the kidneys. [30] An increase in EPO may serve to favourably influence the mitochondrial function of cardiac cells, angiogenesis, cell proliferation, and inflammation, in addition to directly enhancing myocardial tissue oxygen delivery. ${ }^{[30]}$ A recent study demonstrated that erythropoietin levels substantially increase in diabetic patients with coronary artery disease (CAD) after one month on empagliflozin. ${ }^{[30]}$ This is, in addition, to an increase in haematocrit, reduced ferritin, and red blood cell haemoglobin concentrations. Lastly, SGLT2 inhibition has been demonstrated to improve vascular function by inhibiting endothelial cell activation, inducing direct vasorelaxation, reducing endothelial cell dysfunction 
and molecular changes associated with early atherogenesis. [35] The resultant effect is reduced arterial wall stiffness and vascular resistance. Other suggested mechanisms of cardiovascular protection by SGLT2 inhibitors include: blood pressure lowering, weight loss effect, improving glucose control, inhibiting the sympathetic nervous system, inhibiting SGLT1, increasing circulating provascular progenitor cells, and decreasing oxidative stress. [30]

\section{Cardiovascular outcome trials for SGLT2 inhibitors}

As a class, SGLT2 inhibitors have demonstrated a positive effect on CVD and have consistently shown some cardio-protective properties. [36] Initial data suggested that the cardiac benefits of the SGLT2 inhibitors in reducing cardiac ischaemic events were limited to those with preexisting atherosclerotic disease. [37] However, meta-analysis looking at the EMPA-REG, CANVAS and DECLARE-TIMI trials for empagliflozin, canagliflozin and dapagliflozin, respectively revealed an impressive $23 \%$ reduction in $\mathrm{CV}$ deaths and hospitalisation for $\mathrm{HF}$, regardless of pre-existing atherosclerotic disease or HF. [37] Diabetes nephropathy remains a significant health problem. It is the most common cause of end-stage renal disease (ESRD) in the US; it affects approximately $30 \%$ of people with DM. [36] In addition to the CV benefits, SGLT2 inhibitors have demonstrated renal benefits in robust manners. [38,] The trials that highlighted the CV benefits of using SGLT2 inhibitors are discussed below

\section{EMPA-REG Outcome Trial}

The Empagliflozin Cardiovascular Outcome Event Trial in Type 2 Diabetes mellitus PatientsRemoving Excess Glucose (EMPA-REG) was a randomised, double-blinded, placebo-controlled trial consisting of 7,020 participants, $57 \%$ of whom had had T2DM for more than ten years. [39] The average $\mathrm{HbA} 1 \mathrm{c}$ of the study population was $8 \%$, while the mean duration of follow-up was 3.1years. The study which compared the effect of empagliflozin and placebo was an event-driven study that compared the effectiveness and safety of $10 \mathrm{mg}$ and $25 \mathrm{mg}$ empagliflozin, both administered daily with placebo. Patients with high CV risk were enrolled explicitly for this study. The primary endpoint was defined as the time it took for MACE to occur, and this was defined as $\mathrm{CV}$ death, nonfatal MI, or nonfatal stroke. The results showed that T2DM patients with pre-existing CVD treated with empagliflozin showed superior outcomes in MACE in terms of death from CV causes, nonfatal MI, or nonfatal stroke, compared to those given a placebo. [39] The EMPA-REG OUTCOME trial further revealed that empagliflozin reduces the risk of nephropathy compared to the placebo group. It has also shown that it also reduces the progression of macroalbuminuria.

\section{CANVAS TRIAL}

This was a randomised, placebo-controlled trial (to evaluate the CV safety of canagliflozin) in which 10,142 participants with T2DM were followed up for at least ten years (mean duration 13.5 years). [40] This study compared patients treated with canagliflozin $100 \mathrm{mg}$ or $300 \mathrm{mg}$ with placebo in a 1:1:1 randomisation with a median follow-up duration of 2.4 years. Like the EMPAREG, the primary outcome was death from CV event, nonfatal MI and nonfatal stroke. The mean duration of 13.5 years for DM, while the mean $\mathrm{HbA1c}$ was $8.2 \%$. Interestingly, about $65.6 \%$ of the participants had a history of CVD, and like EMPA-REG, canagliflozin also reduced MACE by $14 \%$. Interestingly, T2DM patients with preexisting CVD treated with canagliflozin had a lower risk of $\mathrm{CV}$ events than those given a placebo. ${ }^{[40]}$ However, there was an increased risk of toe, foot and leg amputations in the CANVAS study.

DECLARE-TIMI TRIAL

The Dapagliflozin Effect on CardiovascuLAR Events-Thrombolysis In Myocardial Infarction 
(DECLARE-TIMI) was a double-blind, randomised trial in which T2DM patients with atherosclerotic cardiovascular disease (ASCVD) or multiple risk factors for ASCVD were randomly commenced on dapagliflozin or given a placebo. ${ }^{[41]}$ The study randomised patients to dapagliflozin $10 \mathrm{mg}$ per day or placebo with 4.2 years mean follow-up time. Altogether, the study recruited 17,160 patients with an $\mathrm{HbA1c}$ level of $6.5 \%-12 \%$. It was essentially a primary prevention study because only $41 \%$ of the participants had established CVD, while $59 \%$ had multiple CV risk factors. Remarkably, dapagliflozin met its primary safety endpoint of non-inferiority for MACE but not superiority for MACE. Impressively dapagliflozin demonstrated a reduction in ASCVD risk and a reduced hospitalisation rate for $\mathrm{HF}$ patients compared to the placebo group. The majority of the participants did not experience $\mathrm{HF}$, which shows that dapagliflozin may be beneficial in the primary prevention of HF. ${ }^{[41]}$ Furthermore, the DECLARE-TIMI trial demonstrated a favourable renoprotective effect from dapagliflozin usage. ${ }^{[42]}$

\section{CREDENCE TRIAL}

The CREDENCE trial (Canagliflozin and Renal Outcomes in Type 2 Diabetes and Nephropathy) was the first randomised control trial of an SGLT2 inhibitor specifically designed to evaluate primary kidney outcomes among patients with exclusively albuminuric CKD. ${ }^{[3,44]}$ They enrolled T2DM patients with CKD, who were receiving standard of care, including a maximum tolerated dose of Angiotensin-Converting Enzyme inhibitors (ACEi) or Angiotensin Receptor Blockers (ARB). The patients were randomised to canagliflozin $100 \mathrm{mg}$ daily or placebo and were followed up for 2.6 years, with the trial stopping early for superiority. The primary kidney outcome was defined as a composite of ESRD, doubling serum creatinine, or death from renal or $\mathrm{CV}$ causes. The primary outcome occurred in 43.2 and 61.2 per 1000 patient-years in the canagliflozin and placebo arms, respectively; this translated to a $30 \%$ relative risk reduction in the primary kidney outcome. This study demonstrated that canagliflozin reduces the risk of developing ESRD by 30\%.[37] A secondary analysis of canagliflozin versus CANagliflozin Treatment and Trial Analysis-Sulphonylurea (CANTA-SU) also showed that canagliflozin has a positive effect on renal health. Preclinical and clinical studies have shown that SGLT2 inhibitors have beneficial renal hemodynamic effects, reducing hyperfiltration and intraglomerular pressure, in addition to a positive influence on albuminuria. [44]

\section{DAPA-HF TRIAL}

The Dapagliflozin and Prevention of Adverse Outcomes in Heart Failure (DAPA-HF) trial was designed to determine if the SGLT2 inhibitor dapagliflozin could be used safely and efficaciously in the treatment of patients with HF and reduced ejection fraction $(\mathrm{rEF})$, regardless of diabetes history.[45] A total of 4,744 T2DM patients with $\mathrm{HF}$ and $\mathrm{rEF}$ (irrespective of diabetes status) were randomised to receive dapagliflozin $10 \mathrm{mg}$ daily or a placebo. The results revealed $4.9 \%$ fewer cases of worsening HF or death from CVD for those on dapagliflozin than those on placebo.[45] A significant limitation observed from this study is that enrolled patients were essentially those with moderate HF. Therefore, there is a need for further study in patients with severe HF.

\section{GLUCAGON-LIKE PEPTIDE $\quad-1$ (GLP-1) ANALOGUE}

Sometimes referred to as incretin mimetics, these are potent incretin hormones produced in the Lcells of the distal colon and ileum and are known to have both central and peripheral effects.[46] They reproduce the actions of endogenous GLP1 with the net effect of increasing insulin secretion, inhibiting glucagon production and increasing satiety.[47] Furthermore, they impede glucagon production from pancreatic alpha cells when the blood glucose levels are high. ${ }^{[48]}$ Glucagon, by 
glycogenolysis, stimulates the conversion of liver glycogen to glucose, thereby raising blood glucose. They also delay gastric emptying and can decrease pancreatic beta-cell apoptosis while promoting their proliferation. ${ }^{24]}$ Slowing of gastric emptying maximises nutrient absorption while reducing weight gain. ${ }^{[25]}$ They have been shown to positively affect myocardial contractility, hypertension control (natriuretic/diuretic effect), endothelium (antiatherosclerotic), and lipid profile.[49] An absolute benefit in the lipid parameters (improvement in HDL cholesterol and fasting triglycerides) has been ascribed directly to weight loss. [50]

The figure below shows a summary of the mechanism of action. [44] Table I shows an overview of available GLP-1 RA (Isabel Del Olmo-Garcia and MerinoTorres, 2018)

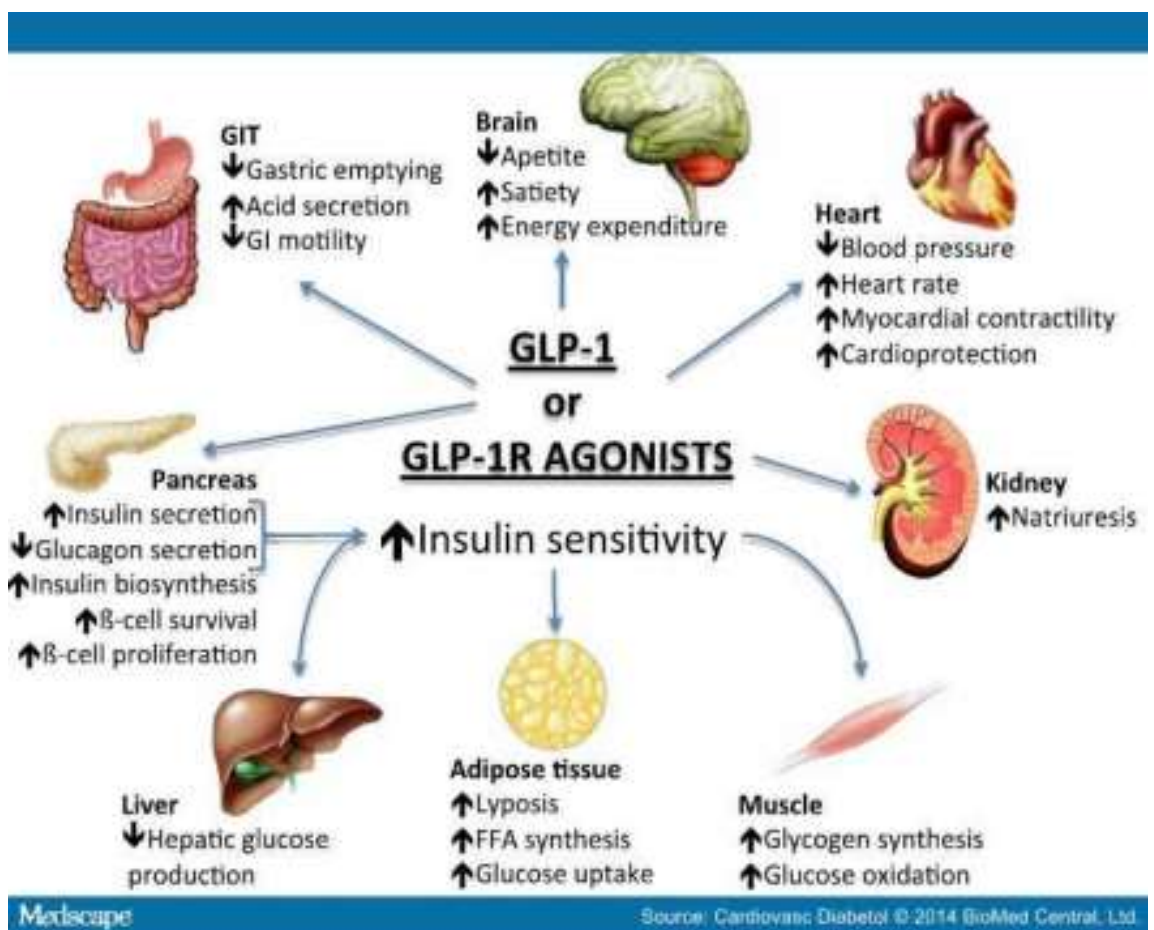

Figure 2: Mechanism of action of GLP-RA

Saraiva, F.K., and Sposito, A.C. 'Cardiovascular Effects of Glucagon-like peptide 1 (GLP1) Receptor Agonists,' Cardiovasc Diabetol 2014; 13(142). Available at: https://www.medscape.com/viewarticle/835947. (Accessed 22 July 2021).

Table I: List of available GLP-1 RA

\begin{tabular}{llll}
\hline GLP-1 RAS & Brand name & Administration & Action \\
\hline Exenatide & Byetta ${ }^{\circledR}$ & Twice daily & Short-acting \\
Exenatide-LAR & Bydureon ${ }^{\circledR}$ & Once weekly & Long-acting \\
Lixisenatide & Lyxumia ${ }^{\circledR}$ & Once-daily & Short-acting \\
Liraglutide & Victoza ${ }^{\circledR}$ & Once-daily & Long-acting \\
Albiglutide & Eperzan ${ }^{\circledR}$ & Once-daily & Long-acting \\
Dulaglutide & Trulicity ${ }^{\circledR}$ & Once-daily & Long-acting \\
\hline
\end{tabular}

Isabel Del Olmo-Garcia, M., \& Merino-Torres, J. F. GLP-1 Receptor Agonists and Cardiovascular Disease in Patients with Type 2 Diabetes 2018. https://doi.org/10.1155/2018/4020492 


\section{Mechanism of cardiovascular protection by GLP-1 analogues}

Just like the SGLT2 inhibitors, their mechanism of $\mathrm{CV}$ risk reduction is not entirely understood. However, several theories have been postulated. They have been shown to improve endothelial functions, though it is unclear if the effect is direct or indirect. ${ }^{[4]}$ Also, their beneficial role in blood pressure and heart rate regulation has been increasingly highlighted. A meta-analysis of 16 RCTs showed that exenatide and liraglutide lowered systolic blood pressure by a mean of 5.24 $\mathrm{mmHg}$ more than placebo and $3.46 \mathrm{mmHg}$ more than insulin glargine. Similarly, they lowered the diastolic pressure by $5.91 \mathrm{mmHg}$ more than placebo and $0.99 \mathrm{mmHg}$ more than glargine. [51] Furthermore, the drugs decrease hepatic production of Very Low-Density Lipoprotein (VLDL) triglycerides by modulating the liver enzymes involved in lipid metabolism. Additionally, they inhibit pro-inflammatory atherogenic mediators. ${ }^{52]}$ Their role in cardioprotection has primarily been shown in animal models with MI, where they reduced the infarct size while preserving left ventricular ejection fraction after an infarct. Remarkably, they protect the cardiomyocyte and endothelial cells from unwanted injuries. [53]

\section{Cardiovascular outcome trials of GLP-1 receptor agonists}

Cardiovascular outcome trials were performed on many GLP-1 receptor agonists as described below. [50]

\section{ELIXA TRIAL}

Evaluation of LIXisenatide in Acute coronary syndrome (ELIXA) trial was the first trial with a GLP-1 analogue aimed at evaluating the effect of the drug lixisenatide in acute coronary syndrome and was published in 2016. [54] Those enrolled in this study were persons with T2DM who also had an acute coronary event within 180 days before screening. The study randomised patients to lixisenatide or a volume-matched placebo. The primary endpoint was the first occurrence of death from CV causes, nonfatal stroke, nonfatal MI, or hospitalisation for unstable angina. They enrolled a total of 6,068 patients with a mean HbA1c of $7.7 \%$ who were followed up for 25 months. The results showed that about $27.5 \%$ of subjects in the lixisenatide arm discontinued the study. Also, more patients in the treatment group reached the primary endpoint compared with the placebo. Furthermore, the percentage change in the urinary ACR was more remarkable in the placebo, though not significant. Additionally, the lixisenatide group had more adverse events when compared with placebo (11.4\% vs $7.2 \%)$.

Unfortunately, this led to the permanent discontinuation of the drug. Another unexpected finding from this study is that the rates of serious adverse events were reassuringly similar in both groups. It is necessary to emphasise that this study was specifically designed to demonstrate non-inferiority rather than superiority. Moreover, it had a relatively shorter follow-up period and had the highest percentage of subjects on statin treatment which confers CV benefit. For reasons not clear, drug compliance was relatively lower when compared with other trials. Interestingly, the improvement in their $\mathrm{HbA1c}$ is remarkable even though their body weight remained unchanged. Therefore, concerning the cardiovascular profile, the drug was scientifically neutral. [55]

\section{LEADER TRIAL}

The Liraglutide Effect and Action in Diabetes: Evaluation of Cardiovascular Outcome Results (LEADER) trial was published in 2016. [56] It began in 2010 with a total of 9,340 participants who had T2DM with increased CV risk. This study sought to demonstrate non-inferiority of liraglutide compared to placebo for $\mathrm{CV}$ risk. It was a randomised, double-blinded, placebocontrolled, multi-centre study. The intervention group received liraglutide $1.8 \mathrm{mg}$ subcutaneously 
daily versus placebo: this served as an add-on to existing therapy, with a median follow up of 3.8 years. The results revealed that established CVD was present in $81.3 \%$ while CKD (>Stage 3) was present in $24.7 \%$; this showed that the study population had a high risk of CV events. Gladly, the incidence of hypoglycaemia was less frequent in the liraglutide arm $(2.4 \%$ vs $3.3 \%, P=0.02)$. On the other hand, some side-effects that could lead to the stoppage of treatment were more common in the liraglutide group $(9.5 \%$ vs $7.3 \%, P<0.001)$. It was the first study that demonstrated the CV benefits of the GLP-1 analogues. At the end of the research and using the time-to-event analysis, it was observed that the rate of occurrence of death from CV causes, nonfatal MI or nonfatal stroke among patients with T2DM was lower with liraglutide than with placebo. ${ }^{[49]}$ Available data also demonstrated significant improvements in glycaemic control, modest weight loss, a modest reduction in systolic $\mathrm{BP}$, and reduced incidence of nephropathy. The strength of this study lies in its relatively large sample size and long study duration compared to other CVOTs. Furthermore, it was well designed with an expanded study area involving several countries and centres; this design could reveal potential environmental confounders.

Also, it appears that few patients dropped out, with $97 \%$ and $96.6 \%$ of liraglutide and placebo subjects completing the trial. However, we cannot overlook some of the practical limitations. Firstly, this was a partly industry-sponsored study that raises some concerns on potential conflicts of interest. Secondly, some subjects did not complete the survey while others discontinued the trial, hence not having any desired outcome. These subjects were removed after their last visit, implying that any event that may have taken place after that visit was not included. This has far-reaching implications as important events could have been missed thus, denying this study of other vital data. Thirdly, the study period of 3.5-5 years was relatively short, meaning that some safety and efficacy data for the long-term will not have been observed. Fourthly, we are not sure of the effect of liraglutide on microvascular outcomes such as retinopathy, neuropathy, and nephropathy since they may take up to 5-10 years or more to develop compared to the average study follow-up of 3.8 years.

Furthermore, it is not clear why this study showed favourable CV outcome results when several previous CVOTs achieved only neutral results. Additionally, the study enrolled subjects with high risk for CVD, meaning that practical benefits and risks may not apply to patients with lower-risk of CVD. In conclusion, the LEADER study has highlighted the immense use of a GLP1 agonist with an excellent $\mathrm{CV}$ risk profile and potentially better safety profile.

\section{SUSTAIN- 6 TRIAL}

The 'Trial to Evaluate Cardiovascular and Other Long-term Outcomes with Semaglutide in Subjects with Type 2 Diabetes' (SUSTAIN-6) was published in 2016. [57] The study enrolled subjects with T2DM who were 50 years of age or older with pre-existing CVD, chronic HF or CKD (Stage 3 or higher), or 60 years of age and older with at least one $\mathrm{CV}$ risk factor. The primary composite outcome was death from CV events, nonfatal MI or nonfatal stroke. They randomised 3,297 patients, with 3232 of them completing the study over a median time of 2.1 years. The results were impressive: similar to the finding in the LEADER study, $83 \%$ of patients had established CVD (including CKD Stage 3), 59\% had established CVD, not including CKD, with mean $\mathrm{HbA1c}$ being $8.7 \%$. The primary composite $\mathrm{CV}$ outcome was significantly lower in the semaglutide arm (6.6\%) than placebo (8.9\%). There was no significant difference in the risk of $\mathrm{CV}$ death or nonfatal MI, although there were significantly fewer nonfatal strokes in the semaglutide arm (1.6\%) than placebo $(2.7 \%)$. Similarly, there were fewer cases of DM retinopathy in the semaglutide arm than in the 
placebo arm $(1.8 \%$ vs $3.0 \%, P=0.02)$. Also, there were significantly lower cases of new or worsening nephropathy in the semaglutide arm than placebo ( $3.8 \%$ vs $6.1 \%, P=0.005)$. This study, just like the LEADER trial, reported higher frequencies of adverse events in the semaglutide arm $(11.5 \%$ vs $5.7 \%)$, but it did not report any difference between both groups.

It is noteworthy to say that this trial had an enormous improvement of $\mathrm{HbA1c}$ with semaglutide when compared with the control $(-0.7 \%$ and $-1.0 \%$ for semaglutide $0.5 \mathrm{mg}$ and $1.0 \mathrm{mg}$ compared with the control), and thus, questioning the source of the observed $\mathrm{CV}$ as coming from the medication itself or the improved glycaemic control. However, we note some limitations of the study. Firstly, the duration of patient follow-up was relatively short (25 months); this could have influenced the study's outcome. Secondly, the number of MACE reported in the semaglutide group was relatively small (108 out of 1648 participants). Thirdly, while the older group of subjects in this study included patients with established CVD and patients with $\mathrm{CV}$ risk factors, only patients with established CVD were recruited among the younger population. This disparity may limit the ability to make assumptions about the consistency of the perceived clinical benefits among the older T2DM subjects with high CV risk. In conclusion, this trial had similar results to the LEADER trial in that, at the end of the study, it was observed that among patients with T2DM and high CV risk, the rate of the first occurrence of death from CV causes, nonfatal MI and nonfatal stroke was significantly lower in the semaglutide group than those receiving placebo. [57]

\section{EXSCEL TRIAL}

This subsequent trial on CV outcomes of GLP-1 analogues was published in 2017. It involved a total of 14,752 randomised patients with an average follow-up of 3.2 years in what is referred to as The Effect of Once-Weekly Exenatide on Cardiovascular Outcomes in T2DM (EXSCEL) trial. ${ }^{[58]}$ The study reported that among patients with T2DM with or without previous risk of CVD, the rate of occurrence of MACE did not differ significantly between patients receiving exenatide and those in the placebo group, thereby conferring it with a neutrality status. ${ }^{[50]} \mathrm{A}$ major limitation of this study was the high discontinuation rates attributed to patients' decision to opt out of the survey. Such a decision appears to be due to the injection device used; it was complex and not patient-friendly. Furthermore, variability may have been introduced because there was no standardisation of the usual-care regimens. In conclusion, the study demonstrated that once-weekly administration of extended-release exenatide in T2DM patients at risk of a CV event appeared not to cause an increase in their overall CV risk.

\section{HARMONY OUTCOMES TRIAL}

This trial, 'Albiglutide and Cardiovascular Outcomes in Patients with Type 2 Diabetes and Cardiovascular Diseases (HARMONY OUTCOMES)', was published in 2018, and it involved 9,463 participants with a study duration of 1.6 years. [59] It evaluated another GLP-1 analogue, albiglutide and CV outcomes in patients with T2DM and CVD. Participants were T2DM patients aged 40 years or more with one of the following criteria: established CVD, cerebrovascular disease or PAD. They were matched at a ratio of $1: 1$ and received subcutaneous injections of albiglutide (which was titrated up to a maximum dose of $50 \mathrm{mg}$ ) or a placebo once a week. The primary outcome was the first occurrence of one of the following: death from $\mathrm{CV}$ causes, MI and stroke. The mean $\mathrm{HbA1c}$ at the commencement of this study was $8.7 \%$, and $24 \%$ of participants in the albiglutide arm prematurely discontinued the medication. The results of the HARMONY trial were impressive; there was a significantly lesser number of patients in the albiglutide arm, which achieved a 
primary composite CV endpoint compared with the placebo arm (7 vs 9\%). Similarly, the decrease in fatal or nonfatal MI in the albiglutide arm was statistically significant.

In contrast to SUSTAIN-6, this study was not designed to assess microvascular outcomes such as retinopathy or nephropathy. Remarkably, there was no observed difference between both arms in terms of serious adverse effects and, this ranked as the trial with the shortest study duration. Although the researchers looked at a high-risk population with a relatively high baseline $\mathrm{HbA1c}$ within a relatively short period, it was a major limitation since the short study duration did not allow for adequate time to evaluate the effect of this drug on long-term microvascular endpoints as well as all-cause mortality. Another notable limitation was the inability to measure serum lipids and urinary albumin excretion, making it challenging to explore other potential mechanisms behind the observed outcomes. In conclusion, the HARMONY OUTCOMES study reported that in patients with T2DM and CVD, albiglutide was superior to placebo concerning major $\mathrm{CV}$ outcomes. [59]

\section{REWIND TRIAL}

This study, named Dulaglutide and Cardiovascular Outcomes in Type 2 Diabetes (REWIND), was published in 2019. It evaluated dulaglutide's superiority (unlike other trials designed to show non-inferiority) and CV outcomes in T2DM patients. [60] The study enrolled a total of 9,901 participants who were 50 years of age and above with T2DM and either a history of previous CV events or CV risk factors. The participants were randomly assigned to dulaglutide $1.5 \mathrm{mg}$ or placebo. The primary outcome was similar to that of other trials, which was the first occurrence of any of the following: nonfatal MI, nonfatal stroke, or death from CV causes or unknown causes. The average baseline HbA1c was $7.2 \%$ (similar to other trials) with a relatively longer median follow-up of 5.4 years. Of all the participants, $31.5 \%$ had previous CVD, lower than $83 \%$ recorded in the LEADER trial. The primary $\mathrm{CV}$ composite outcome occurred in $12 \%$ of participants in the dulaglutide group compared with $13.4 \%$ in the placebo group, which was significant. In the secondary analysis, nonfatal stroke was significantly lower in the dulaglutide group compared with placebo, in addition to significantly fewer renal outcomes. However, there were no differences in eye outcomes and the rate of serious adverse events. Remarkably, this study was different from previous CVOTs. It was the most extended trial with the lowest risk population (with only $32 \%$ of participants having underlying CVD) and the lowest baseline $\mathrm{HbA1c}$. In conclusion, the REWIND trial reported that dulaglutide has clear $\mathrm{CV}$ benefits and could be considered for managing T2DM in middle-aged and older people who have either CVD or CV risk factors. [60]

\section{PIONEER 6 TRIAL}

The trial, published in 2019, was the last and the most recent on CV outcomes of GLP-1 analogues. [61] It evaluated the safety profile of oral semaglutide compared with placebo among T2DM patients with high CV risk. Although safety has been established for subcutaneous semaglutide, but not for oral semaglutide. A randomised, parallel, placebo-controlled, blinded study involved 3,183 T2DM patients with high CV risk who were randomised to oncedaily oral semaglutide to reach a target dose of $14 \mathrm{mg}(\mathrm{n}=1,591)$ or placebo $(\mathrm{n}=1,592)$. The mean duration of follow-up was 15.9 months, while the mean patient age was 66 years. The inclusion criteria included age at least 50 years with established CV risk or CKD or age at least 60 years or older with $\mathrm{CV}$ risk factors only. Expectedly, the results revealed that the primary outcome, CV death, MI, or stroke, occurred in $3.8 \%$ of the semaglutide group compared with $4.8 \%$ in the placebo group. This implies that the 
$\mathrm{CV}$ risk profile of the oral drug semaglutide was not inferior to that of placebo. [61 In conclusion, the PIONEER 6 trial demonstrated robust $\mathrm{CV}$ benefit of oral semaglutide in middle-aged or elderly T2DM patients with high CV risk or CKD.

Table II: Comparison of anti-diabetic agents in the CVOTs and their cardiovascular benefits

\begin{tabular}{|c|c|c|c|c|c|c|c|c|}
\hline Drug & Trial name/year & No of participants & $\begin{array}{l}\text { \% with } \\
\text { CVD }\end{array}$ & $\begin{array}{l}\text { Follow up } \\
\text { duration } \\
\text { (years) }\end{array}$ & $\begin{array}{l}\text { Average } \\
\text { A1c }(\%)\end{array}$ & $\begin{array}{l}\text { Primary } \\
\text { outcome }\end{array}$ & Kidney outcome & Adverse effects \\
\hline \multicolumn{9}{|c|}{ SGLT2 inhibitors } \\
\hline Empagliflozin & EMPA-REG & 7020 & 100 & 3.1 & 8.0 & $\downarrow \mathrm{MACE}$ & $\begin{array}{l}\downarrow \text { Incidence/worseni } \\
\text { ng nephropathy }\end{array}$ & $\begin{array}{l}\text { Genital mycotic, } \\
\text { DKA }\end{array}$ \\
\hline Canagliflozin & $\begin{array}{l}\text { CANVAS } \\
\text { CREDENCE }\end{array}$ & 10142 & 65.6 & $\begin{array}{l}2.4 \\
2.6\end{array}$ & 8.2 & $\downarrow \mathrm{MACE}$ & $\begin{array}{l}\downarrow \\
\downarrow \text { ESKD }\end{array}$ & $\begin{array}{l}\uparrow \quad \text { risk } \\
\text { amputations }\end{array}$ \\
\hline Dapagliflozin & DAPA-HF & 4744 & 41 & 4.2 & $6.5-12$ & $\downarrow \mathrm{MACE}$ & $\begin{array}{l}\text { Favourable reno- } \\
\text { protective effect }\end{array}$ & $\begin{array}{l}\text { Genital mycotic, } \\
\text { DKA }\end{array}$ \\
\hline \multicolumn{9}{|c|}{ GLP-1 receptor agonists } \\
\hline Lixisenatide & ELIXA-2016 & 6068 & $>80.0$ & 2.1 & 7.7 & Neutral & $\downarrow$ & None \\
\hline Liraglutide & LEADER-2016 & 9340 & 81.3 & 3.8 & 8.7 & $\downarrow \mathrm{MACE}$ & $\downarrow$ & GI \\
\hline Semaglutide & SUSTAIN 6-2016 & 3232 & 81.3 & 2.1 & 8.7 & $\downarrow \mathrm{MACE}$ & $\begin{array}{l}\downarrow \text { Incidence/ worseni } \\
\text { ng nephropathy }\end{array}$ & GI \\
\hline Exenatide & EXSCEL-2017 & 14752 & 73.0 & 3.2 & 8.0 & Neutral & Neutral & None \\
\hline Albiglutide & $\begin{array}{l}\text { HARMONY- } \\
2018\end{array}$ & 9463 & & 1.6 & 8.7 & $\downarrow \mathrm{MACE}$ & Neutral & $\begin{array}{l}\text { Injection site } \\
\text { reaction }\end{array}$ \\
\hline $\begin{array}{l}\text { Semaglutide } \\
\text { (Oral) }\end{array}$ & $\begin{array}{l}\text { PIONEER 6- } \\
2019\end{array}$ & 3183 & & 1.3 & & Neutral & NA & GI \\
\hline Dulaglutide & REWIND-2019 & 9901 & 31.5 & 5.4 & 7.2 & $\downarrow \mathrm{MACE}$ & $\downarrow$ & GI \\
\hline
\end{tabular}

\section{Discussion}

Recent data has shown that the new anti-diabetic drug classes, such as GLP-1 RAs and SGLT-2 inhibitors, have significant $\mathrm{CV}$ benefits. These benefits justify their inclusion in several guidelines that recommended them to manage T2DM patients with ASCVD. ${ }^{[16,49]}$ However, our general observations include their CV benefit, which is predicated on lowering the risk of MACE, hospitalisation for HF, and renal events. Furthermore, SGLT-2 inhibitors reduced CV and all-cause mortality, hospitalisation for HF, and renal events the most among the two drug classes. [49] In the LEADER and EMPA-REG OUTCOME trials, liraglutide and empagliflozin were reported to reduce $\mathrm{CV}$ events, CV mortality, and all-cause mortality, respectively. [62] Remarkably, more CVOTs have been completed and published within the last five years, and this reflects the importance given to these classes of drugs in the management of T2DM and its complications in recent times.

The results from EXSCEL and CANVAS trials have not only confirmed the CV safety profiles of GLP-1 RAs and SGLT-2 inhibitors but caused a paradigm shift in the management of ASCVDs. Going further, we still observed some unique differences in $\mathrm{CV}$ outcomes between some of the studies. For instance, the superiority seen with albiglutide, a GLP-1 RA in decreasing MACE was also seen in HARMONY Outcomes. ${ }^{[59]}$ However, no significant reduction in MACE was observed with dapagliflozin and in DECLARE-TIMI, even though the study confirmed its benefit in reducing HF hospitalisation. [41] This contrasted with findings from the EMPA-REG OUTCOME and CANVAS trials. The reasons for this disparity are unclear. However, differences in patients' characteristics and drug use modalities across trials could provide plausible 
explanations. More than half of the T2DM patients enrolled in DECLARE-TIMI did not have CVD but only had risk factors. ${ }^{[41]}$ On the contrary, almost all the patients included in EMPAREG OUTCOME, and more than half of the patients included in CANVAS had diabetes with established CVD. [39] Therefore, it was more challenging to demonstrate a reduction in MACE in patients without pre-existing CVD, as seen in DECLARE-TIMI. Moreover, it is possible that dapagliflozin, used in this trial, has less protection against MACE compared to empagliflozin and canagliflozin. Nevertheless, the reduction in HF hospitalisation with dapagliflozin was the same across several subgroups irrespective of history of ASCVD, HF and CKD. [41]

Another observation is the difference between the results seen in the CANVAS study with what was reported in the EMPA-REG study. Though the EMPA-REG reported a significant decrease in $\mathrm{CV}$ events and all-cause mortality, this substantial decrease was not observed in CANVAS. ${ }^{\left[{ }^{40]} \mathrm{Also}\right.}$, the reasons for this difference remains unclear. However, we can attribute this to the unique characteristics of these patients; about one-third of the patients in the CANVAS trial were in primary prevention, unlike in EMPA-REG, where almost all the patients were in secondary prevention.

Furthermore, data from two recent CVOTs included in the meta-analysis reported similar protection against MACE by GLP-1 RAs and SGLT-2 inhibitors. It is noteworthy to highlight the added renal benefits of these drug classes, just as we have seen in CV studies. ${ }^{[44]}$ Renal diseases are frequently seen in DM patients, and they are associated with increased morbidity and mortality. It has been argued that these drugs may potentially improve renal outcomes in patients with T2DM due to their profound renal and CV benefits, just as was demonstrated in the CREDENCE trial. [44]. It is interesting to note that there is yet to be a comparative CVOT study to determine the superiority of one drug over another in terms of cardiovascular benefits. The inexorable benefits of these drugs are known; however, we are concerned that apart from the LEADER and REWIND trials, which had South Africa as enrolment site, all the studies were done outside sub-Saharan Africa. This has implications. Firstly, we cannot infer the effects of these drugs in black Africans since they were initially tested on patients of European, Asian and American descent. For instance, we still have not forgotten the different responses of Blacks versus Caucasians to angiotensin-converting enzyme inhibitors and angiotensin receptor blockers.

Therefore, there is a need to design similar studies tailored for sub-Saharan African cohorts to assess the effect of these drugs on a different patient population. Furthermore, we note the relatively large sample sizes of these studies even when the inclusion criteria entail far-reaching cardiovascular work-up in addition to the meticulous patient data storage for several years; it reflects the commitment of both the investigators, regulatory authorities and patient population. This is an area where an improvement is needed in our clime because of the challenges we face in electronic data storage and patient follow-up. Additionally, we note the enormous cost of funding such extensive multicentre studies; hence, this is another area where much help is needed. It is worrisome that most drug companies focus their trials on North America, Europe and Asia, but only resort to marketing such drugs in Africa after getting regulatory approvals. This calls for concerted efforts by stakeholders in sub-Saharan Africa and Nigeria to explore all available options of convincing these multinational drug companies on the need to replicate such studies in our clime. More importantly, there is a need to lower the cost of these drugs, which are currently prohibitive and not affordable for the average 
patient in Africa, where poverty and lack of healthcare insurance are prevalent. Therefore, sub-Saharan Africa should be granted special pricing consideration because of its peculiar patient characteristics and challenges.

\section{Conclusion}

SGLT2 inhibitors confer dramatic beneficial cardiovascular risk reduction on patients with T2DM. The various trials have shown the remarkable role of SGLT2 inhibitors in ensuring glycaemic control and cardiovascular risk reduction. Adopting SGLT2 inhibitors as the second-line to metformin is in the right direction for patients with T2DM. It can also be concluded from the discussion above that GLP-1 analogues have emerged as an essential class of medications that we need to consider in the management of T2DM. In addition, making inferences from the seven trials summarised above concerning the cardiovascular outcomes of these agents, it can be inferred that the drugs can help reduce cardiovascular risks in T2DM patients. With the evidence available from the different clinical trials and meta-analyses, SGLT2 inhibitors and GLP-1 analogues have proven to be effective in reducing the $\mathrm{CV}$ risk factors and the death rates associated with T2DM and also, reduce major cardiovascular events. Though these agents have been proven by evidence to be beneficial in managing T2D with added positive $\mathrm{CV}$ outcomes, the high cost makes them less accessible to the average diabetic patient, especially in a resource-poor country such as Nigeria. Measures should be put in place to make these medications available to those who benefit from their use.

Acknowledgement: We thank the staff of the Department of Medicine, Federal Medical Centre, Umuahia, University of Benin Teaching Hospital Benin City and College of Medicine, Bingham University, Karu, Nigeria, who made substantial contributions to the conception and revision of the important intellectual contents of the manuscript.

Authors' Contributions: EIU conceived and designed the study, did a literature review, data extraction, and drafted the manuscript. EA and OEA participated in drafting the manuscript and review of the manuscript for sound intellectual contents. All the authors approved the final version of this manuscript.

Conflicts of Interest: None.

Funding: Self-funded.

Publication History: Submitted 03 May 2021; Accepted 04 August 2021.

\section{References}

1. Saeedi P, Petersohn I, Salpea P, Malanda B, Karuranga S, Unwin N; IDF Diabetes Atlas Committee. Global and regional diabetes prevalence estimates for 2019 and projections for 2030 and 2045: Results from the International Diabetes Federation Diabetes Atlas, 9th edition. Diabetes Res Clin Pract 2019; $\quad 157$ : 107843. https://doi.org/10.1016/j.diabres.2019.1078 $\underline{43}$

2. Diabetes. World Health Organization: 2020 Diabetes. Available at: https://www.who.int/healthtopics/diabetes\#tab=tab_1 Accessed on 08 April 2021.

3. Colberg SR, Sigal RJ, Fernhall B, Regensteiner JG, Blissmer BJ, Rubin RR, et al.; American College of Sports Medicine; American Diabetes Association. Exercise and Type 2 Diabetes: The American College of Sports Medicine and the American Diabetes Association: Joint Position Statement. Diabetes Care 2010; 33: e147-167. https://doi.org/10.2337/dc10-9990

4. Ajala O, English P, Pinkney J. Systematic review and meta-analysis of different dietary approaches to the management of Type 2 Diabetes. Am J Clin Nutr 2013; 97: 505-516. https://doi.org/10.3945/ajcn.112.042457 
5. Madden KM. Evidence for the benefit of exercise therapy in patients with type 2 diabetes. Diabetes Metab Syndr Obes 2013; 6: 233-239.

https://doi.org/10.2147/DMSO.S32951

6. Kalofoutis C, Piperi C, Kalofoutis A, Harris F, Phoenix D, Singh J. Type II diabetes mellitus and cardiovascular risk factors: Current therapeutic approaches. Exp Clin Cardiol 2007; $12: 17-28$.

7. Kannel W, McGee D. Diabetes and glucose tolerance as risk factors for cardiovascular disease: The Framingham study. Diabetes Care 1979; 2: 120-126. https://doi.org/10.2337/diacare

8. Rosengren A, Hagman M, Wedel H, Wilhelmsen L. Serum cholesterol and longterm prognosis in middle-aged men with myocardial infarction and angina pectoris. A 16-year follow-up of the Primary Prevention Study in Göteborg, Sweden. Eur Heart J 1997; 18:

754-761.

https://doi.org/10.1093/oxfordjournals.eur heartj.a015340

9. Haffner SM, Lehto S, Rönnemaa T, Pyörälä K, Laakso M. Mortality from coronary heart disease in subjects with type 2 diabetes and in nondiabetic subjects with and without prior myocardial infarction. N Engl J Med 1998; 339:

229-234.

https://doi.org/10.1056/NEJM19980723339 $\underline{0404}$

10. Morrish NJ, Wang SL, Stevens LK, Fuller JH, Keen $\mathrm{H}$. Mortality and causes of death in the WHO Multinational Study of Vascular Disease in Diabetes. Diabetologia 2001; 44 Suppl 2: S14-S21. https://doi.org/10.1007/pl00002934

11. Joshi SS, Singh T, Newby DE, Singh J. Sodium-glucose co-transporter 2 inhibitor therapy: mechanisms of action in heart failure. Heart 2021. https://doi.org/10.1136/heartinl-2020$\underline{318060}$
12. Abdul-Ghani M, DeFronzo RA, Del Prato S, Chilton R, Singh R, Ryder REJ. Cardiovascular Disease and Type 2 Diabetes: Has the Dawn of a New Era Arrived? Diabetes Care 2017; 40: 813-820. https://doi.org/10.2337/dc16-2736

13. Leon, B. Diabetes and cardiovascular disease: Epidemiology, biological mechanisms, treatment recommendations and future research. World J Diabetes 2015; 6: 1246. https://doi.org/10.4239/wjd.v6.i13.1246

14. Subramanian S, Hirsch I. Personalised diabetes management: Moving from algorithmic to individualised therapy. Diabetes Spectrum 2014; 27: 87-91. https://doi.org/10.2337/diaspect.27.2.87

15. Kulzer B, Daenschel W, Daenschel I, Schramm W, Messinger D, Weissmann J, et al. Integrated personalised diabetes management improves glycemic control in patients with insulin-treated type 2 diabetes: Results of the PDM-ProValue study program. Diabetes Res Clin Pract 2018; 144: 200-212. https://doi.org/10.1016/j.diabres.2018.09.00 2

16. U.S Food and Drug Administration. Endocrinologic and Metabolic Drugs Advisory Committee Meeting 2018. Available at:

https://www.fda.gov/media/121272/down load Accessed 31 March 2021.

17. Abdul-Ghani M, Jayyous A, Asaad N, Helmy S, Al-Suwaidi J. Pioglitazone and cardiovascular risk in T2DM patients: is it good for all? Ann Transl Med 2018; 6: 192. https://doi.org/10.21037/atm.2018.03.19

18. Liao HW, Saver JL, Wu YL, Chen TH, Lee M, Ovbiagele B. Pioglitazone and cardiovascular outcomes in patients with insulin resistance, pre-diabetes and type 2 diabetes: a systematic review and meta-analysis. BMJ Open 2017; 7: e013927. https://doi.org/10.1136/bmjopen2016-013927 
19. Filion KB, Douros A, Azoulay L, Yin H, Yu $\mathrm{OH}$, Suissa S. Sulfonylureas as initial treatment for type 2 diabetes and the risk of adverse cardiovascular events: A populationbased cohort study. Br J Clin Pharmacol 2019; 85: 2378-2389. https://doi.org/10.1111/bcp.14056

20. Phung OJ, Schwartzman E, Allen RW, Engel SS, Rajpathak SN. Sulphonylureas and risk of cardiovascular disease: systematic review and meta-analysis. Diabet Med 2013; 30: 11601171. https://doi.org/1 0.1111/dme.12232

21. Schernthaner G, Karasik A, Abraitienè A, Ametov AS, Gaàl Z, Gumprecht J, et al. Evidence from routine clinical practice: EMPRISE provides a new perspective on CVOTs. Cardiovasc Diabetol 2019; 18: 115. https://doi.org/10.1186/s12933-019-0920-3

22. Food and Drug Administration. 'SGLT-2 inhibitors' 2018. Available at https://www.fda.gov/drugs/postmarketdrug-safety-informationpatientsandproviders/sodium-glucosecotransporter-2-sglt2-inhibitors Accessed 31 March 2021.

23. Bond A. Exenatide (Byetta) as a novel treatment option for type 2 diabetes mellitus. Proc (Bayl Univ Med Cent) 2006; 19: 281-294. https://doi:10.1080/08998280.2006.11928181

24. Collins L, Costello R. Glucagon-like Peptide1 Receptor Agonists 2020. Available at: https://www.ncbi.nlm.nih.gov/books/NBK 551568/ Accessed 08 April 2021.

25. Kanai Y, Lee WS, You G, Brown D, Hediger MA. The human kidney low-affinity $\mathrm{Na}+$ /glucose co-transporter SGLT2. Delineation of the major renal reabsorptive mechanism for D-glucose. J Clin Invest 1994; 93:

397-404.

https://doi.org/10.1172/JCI116972
26. Nomura S. Renal Threshold Case Histories in Recent Drug Discovery 2017. Available at: https://www.sciencedirect.com/topics/me dicine-and-dentistry/renal-threshold Accessed 10 April 2021.

27. Hsia DS, Grove O, Cefalu WT. An update on sodium-glucose co-transporter-2 inhibitors for the treatment of diabetes mellitus. Curr Opin Endocrinol Diabetes Obes 2017; 24: 7379.

https://doi.org/10.1097/MED.000000000000 $\underline{0311}$

28. Cornell, S. Mode of action of SGLT2 inhibitors in the kidney. 2015. Available at: https://www.researchgate.net/figure/Mod e-of-action-of-SGLT2-inhibitors-in-thekidneyCopiedwith-permission-from-Scheen12_fig1_283449175 Accessed 22 July 2021.

29. Verma S, McMurray J. SGLT2 inhibitors and mechanisms of cardiovascular benefit: a stateof-the-art review. Diabetologia 2018; 61: 2108-2117. https://doi.org/10.1007/s00125018-4670-7

30. Lopaschuk G, Verma S. Mechanisms of Cardiovascular Benefits of Sodium-Glucose Co-Transporter 2 (SGLT2) Inhibitors. JACC Basic Transl Sci 2020; 5: 632-644. https://doi.org/10.1016/j.jacbts.2020.02.004

31. Santos-Gallego CG, Requena-Ibanez JA, San Antonio R. Empagliflozin ameliorates adverse left ventricular remodelling in nondiabetic heart failure by enhancing myocardial energetics. J Am Coll Cardiol 2019; 73: 1931-1944.

32. Iborra-Egea O, Santiago-Vacas E, Yurista SR. Unraveling the molecular mechanism of action of empagliflozin in heart failure with reduced ejection fraction with or without diabetes. J Am Coll Cardiol Basic Trans Science 2019; 4: 831-840.

33. Chino Y, Samukawa Y, Sakai S. SGLT2 inhibitor lowers serum uric acid through alteration of uric acid transport activity in 
renal tubule by increased glycosuria. Biopharm Drug Dispos 2014; 35: 391-404.

34. Nussenzweig SC, Verma S, Finkel T. The role of autophagy in vascular biology. Circ Res 2015; 116: 480-488.

35. Juni RP, Kuster DWD, Goebel M, Helmes M, musters R, van der Velden J, et al. Cardiac microvascular endothelial enhancement of cardiomyocyte function is impaired by inflammation and restored by empagliflozin. J Am Coll Cardiol Basic Trans Science 2019; 4: 575-591.

36. Zelniker TA, Wiviott SD, Raz I, Im K, Goodrich EL, Bonaca MP, et al. SGLT2 inhibitors for primary and secondary prevention of cardiovascular and renal outcomes in type 2 diabetes: a systematic review and meta-analysis of cardiovascular outcome trials. Lancet 2019; 393: 31-39. https://doi.org/10.1016/S0140-

6736(18)32590-X Erratum in: Lancet 2019; 393(10166): 30.

37. Rabizadeh S, Nakhjavani M, Esteghamati A. Cardiovascular and Renal Benefits of SGLT2 Inhibitors: A Narrative Review. Int J Endocrinol Metab 2019; 17: e84353. https://doi.org/10.5812/ijem.84353

38. Giorgino F, Vora J, Fenici P, Solini A. Renoprotection with SGLT2 inhibitors in type 2 diabetes over a spectrum of cardiovascular and renal risk. Cardiovasc Diabetol 2020; 19: 196. https://doi.org/10.1186/s12933-020$\underline{01163-9}$

39. Zinman B, Wanner C, Lachin JM, Fitchett D, Bluhmki E, Hantel S, et al.; EMPA-REG OUTCOME Investigators. Empagliflozin, Cardiovascular Outcomes, and Mortality in Type 2 Diabetes. N Engl J Med. 2015; 373: 2117-2128. https://doi.org/10.1056/NEJMoa1504720

40. Neal B, Perkovic V, Mahaffey KW, de Zeeuw D, Fulcher G, Erondu ?? et al.; CANVAS Program Collaborative Group. Canagliflozin,
Cardiovascular and Renal Events in Type 2 Diabetes. N Engl J Med 2017; 377: 644-657. https://doi.org/10.1056/NEJMoa1611925

41. Wiviott SD, Raz I, Bonaca MP, Mosenzon O, Kato ET, Cahn A, et al; DECLARE-TIMI 58 Investigators. Dapagliflozin and Cardiovascular Outcomes in Type 2 Diabetes. N Engl J Med 2019; 380: 347-357. https://doi.org/10.1056/NEJMoa1812389

42. El Mouhayyar C, Riachy R, Khalil AB, Eid A, Azar S. SGLT2 Inhibitors, GLP-1 Agonists, and DPP-4 Inhibitors in Diabetes and Microvascular Complications: A Review. Int J Endocrinol 2020; 2020:1762164. https://doi.org/10.1155/2020/1762164

43. Batuman V. What is the role of SGLT2 inhibitors in the treatment of diabetic nephropathy? 2019. Available at: https://www.medscape.com/answers/2389 46-42580/what-isthe-role-of-sglt2-inhibitorsin-the-treatment-of-diabetic-nephropathy Accessed 08 April 2021.

44. Jardine MJ, Mahaffey KW, Neal B, Agarwal R, Bakris GL, Brenner J, et al. The Canagliflozin and Renal Endpoints in Diabetes with Established Nephropathy Clinical Evaluation (CREDENCE) Study Rationale, Design, and Baseline Characteristics. Am J Nephrol 2017; 46: 462-472. $\underline{\text { https://doi.org/10.1159/000484633 }}$

45. McMurray JJV, DeMets DL, Inzucchi SE, Kober L, Kosiborod MN, Martinez FA, et al. A trial to evaluate the effect of the sodiumglucose co-transporter 2 inhibitor dapagliflozin on morbidity and mortality in patients with heart failure and reduced left ventricular ejection fraction (DAPA-HF). Eur J Heart Fail 2019; 21: 665-675. https://doi.org/10.1002/ejhf.1432

46. MacDonald PE, El-Kholy W, Riedel MJ, Salapatek AM, Light PE, Wheeler MB. The multiple actions of GLP-1 on the process of glucose-stimulated insulin secretion. Diabetes 2002;51 Suppl 3: S434-S442. 
https://doi.org/10.2337/diabetes.51.2007.s4 $\underline{34}$

47. Bonora E, DeFronzo A. Diabetes, Epidemiology, Genetics, Pathogenesis, Diagnosis, Prevention, and Treatment.' London: Springer Reference 2018.

48. Iorga RA, Bacalbasa N, Carsote M, Bratu OG, Stanescu AMA, Bungau S, et al. Metabolic and cardiovascular benefits of GLP-1 agonists, besides the hypoglycemic effect (Review). Exp Ther Med 2020;20 : 2396-2400. https://doi.org/10.3892/etm.2020.8714

49. Saraiva F, Sposito A. Cardiovascular Effects of Glucagon-like peptide 1 (GLP1) Receptor Agonists. Cardiovasc Diabetol 2014; 13. Available at: https://www.medscape.com/viewarticle/8 35947 Accessed 08 April 2021.

50. Drucker DJ. Mechanisms of Action and Therapeutic Application of Glucagon-like Peptide-1. Cell Metab 2018; 27: 740-756. https://doi.org/10.1016/j.cmet.2018.03.001

51. Wang B, Zhong J, Lin H, Zhao Z, Yan Z, He $\mathrm{H}, \mathrm{Ni} Y$, et al. Blood pressure-lowering effects of GLP-1 receptor agonists exenatide and liraglutide: a meta-analysis of clinical trials. Diabetes Obes Metab 2013; 15: 737-749. https://doi.org/10.1111/dom.12085

52. Patel VJ, Joharapurkar AA, Shah GB, Jain MR. Effect of GLP-1 based therapies on diabetic dyslipidemia. Curr Diabetes Rev 2014; 10: 238-250.

https://doi.org/10.2174/15733998106661407 $\underline{07092506}$

53. Li J, Zheng J, Wang S, Lau HK, Fathi A, Wang Q. Cardiovascular Benefits of Native GLP-1 and its Metabolites: An Indicator for GLP-1Therapy Strategies. Front Physiol 2017; 8: 15. https://doi.org/10.3389/fphys.2017.00015

54. Sheahan KH, Wahlberg EA, Gilbert MP. An overview of GLP-1 agonists and recent cardiovascular outcomes trials. Postgrad Med
J 2020; 96: 156-161. https://doi.org/10.1136/postgradmedj2019-137186

55. Pfeffer MA, Claggett B, Diaz R, Dickstein K, Gerstein HC, Køber LV, et al.; ELIXA Investigators. Lixisenatide in Patients with Type 2 Diabetes and Acute Coronary Syndrome. N Engl J Med 2015; 373: 2247-2257. https://doi.org/10.1056/NEJMoa1509225

56. Marso S, Gilbert HD, Brown-Frandsen K, Kristensen P, Johannes FE, Nauck MA, et al. Liraglutide and Cardiovascular Outcomes in Type 2 Diabetes. N Engl J Med 2016; 375: 311-322.

https://doi.org/10.1056/NEJMoa1603827

57. Marso SP, Bain SC, Consoli A, Eliaschewitz FG, Jódar E, Leiter LA, Lingvay I, et al.; SUSTAIN-6 Investigators. Semaglutide and Cardiovascular Outcomes in Patients with Type 2 Diabetes. N Engl J Med. 2016; 375: 1834-1844.

https://doi.org/10.1056/NEJMoa1607141

58. Holman RR, Bethel MA, Mentz RJ, Thompson VP, Lokhnygina Y, Buse JB, et al.; EXSCEL Study Group. Effects of Once-Weekly Exenatide on Cardiovascular Outcomes in Type 2 Diabetes. N Engl J Med 2017; 377: 1228-1239.

https://doi.org/10.1056/NEJMoa1612917

59. Hernandez AF, Green JB, Janmohamed $S$, D'Agostino RB Sr, Granger CB, Jones NP, et al.; Harmony Outcomes committees and investigators. Albiglutide and cardiovascular outcomes in patients with type 2 diabetes and cardiovascular disease (Harmony Outcomes): a double-blind, randomised placebocontrolled trial. Lancet 2018; 392: 1519-1529. https://doi.org/10.1016/S0140$\underline{6736(18) 32261-X}$

60. Gerstein HC, Colhoun HM, Dagenais GR, Diaz R, Lakshmanan M, Pais P, et al.; REWIND Investigators. Dulaglutide and cardiovascular outcomes in type 2 diabetes (REWIND): a double-blind, randomised 
placebo-controlled trial. Lancet 2019; 394(10193): $\quad$ 121-130.

https://doi.org/10.1016/S01406736(19)31149-3

61. Husain M. Birkenfeld AL, Donsmark M, Dungan K, Eliaschewitz FG, Franco DR, et al. Oral Semaglutide and Cardiovascular Outcomes in Patients with Type 2 Diabetes', $N$ Engl J Med 2019; 381: 841-851. https://doi.org/10.1056/NEJMoa1901118
62. Brown-Frandsen K, Emerson SS, McGuire DK, Pieber DR, Poulter NR, Pratley RE, et al. Lower rates of cardiovascular events and mortality associated with liraglutide use in patients treated with basal insulin: A DEVOTE sub-analysis. Diabetes Obes Metab 2019;

21:

1437-1444.

https://doi.org/10.1111/dom.13677 source is adequately cited and credited. 